\title{
Is Plant Fitness Proportional to Seed Set? An Experiment and a Spatial Model
}

\author{
Diane R. Campbell, ${ }^{1,2, \star}$ Alison K. Brody, ${ }^{2,3}$ Mary V. Price, ${ }^{2,4}$ Nickolas M. Waser, ${ }^{2,4}$ \\ and George Aldridge ${ }^{2}$
}

1. Department of Ecology and Evolutionary Biology, University of California, Irvine, California 92697; 2. Rocky Mountain Biological Laboratory, Crested Butte, Colorado 81224; 3. Department of Biology, University of Vermont, Burlington, Vermont 05405; 4. School of Natural Resources and Environment, University of Arizona, Tucson, Arizona 85719

Submitted February 13, 2017; Accepted May 30, 2017; Electronically published September 27, 2017

Online enhancements: appendixes. Dryad data: http://dx.doi.org/10.5061/dryad.6q023.

AвSTRACT: Individual differences in fecundity often serve as proxies for differences in overall fitness, especially when it is difficult to track the fate of an individual's offspring to reproductive maturity. Using fecundity may be biased, however, if density-dependent interactions between siblings affect survival and reproduction of offspring from high- and low-fecundity parents differently. To test for such densitydependent effects in plants, we sowed seeds of the wildflower Ipomopsis aggregata (scarlet gilia) to mimic partially overlapping seed shadows of pairs of plants, one of which produced twice as many seeds. We tested for differences in offspring success using a genetic marker to track offspring to flowering multiple years later. Without density dependence, the high-fecundity parent should produce twice as many surviving offspring. We also developed a model that considered the geometry of seed shadows and assumed limited survivors so that the number of juvenile recruits is proportional to the area. Rather than a ratio of $2: 1$ offspring success from high- versus low-fecundity parents, our model predicted a ratio of $1.42: 1$, which would translate into weaker selection. Empirical ratios of juvenile offspring and of flowers produced conformed well to the model's prediction. Extending the model shows how spatial relationships of parents and seed dispersal patterns modify inferences about relative fitness based solely on fecundity.

Keywords: density dependence, fitness, Ipomopsis aggregata, seed set, seed shadow, seedling emergence.

\section{Introduction}

Fitness depends on survival and fertility at each age or stage of the life cycle. A rich theoretical literature relates overall measures of individual fitness, such as the total contribution of offspring or the finite rate of increase, to these ageor stage-specific components (McGraw and Caswell 1996;

\footnotetext{
* Corresponding author; e-mail: drcampbe@uci.edu. ORCIDs: Campbell, http://orcid.org/0000-0002-1147-846X; Price, http:// orcid.org/0000-0002-2223-7792.
}

Am. Nat. 2017. Vol. 190, pp. 818-827. (C) 2017 by The University of Chicago. 0003-0147/2017/19006-57558\$15.00. All rights reserved. DOI: $10.1086 / 694116$
Shaw et al. 2008; Moorad 2014). Estimating overall fitness is, however, challenging under natural conditions. It is not surprising, then, that most empirical studies of natural selection on a trait rely on measuring a component of individual fitness rather than a more integrated measure (98\% of studies according to Kingsolver et al. 2012). For example, in flowering plants, the majority of phenotypic selection estimates on floral traits are based on pollen import, fruit production, or seed production (review in Harder and Johnson 2009), with a smaller number also using an estimate of male fecundity (e.g., Stanton et al. 1986; Conner et al. 1996; Meléndez-Ackerman and Campbell 1998).

A trait can, however, have multiple fitness effects that extend even beyond the portion of the life cycle when the trait is expressed. For example, fecundity need not capture all of the fitness effects of a reproductive trait, which can include viability earlier in the life cycle (Mojica and Kelly 2010). Thus, many investigators have also examined fitness effects of floral traits that occur outside of the time window of pollination and seed maturation. Some such studies of selfcompatible species have examined the influence of traits, such as floral display size, not only on selfing rate during seed formation but also on expression of inbreeding depression in offspring (review by Barrett and Harder 1996). Other studies have examined pleiotropic effects of floral traits on defense against herbivores (Strauss and Whittall 2006; Kessler et al. 2013).

One stage of the life cycle has been almost completely ignored in estimating individual fitness in plants, and that is the difficult-to-characterize transition between seed input to the soil and seedling emergence. Even in cases where investigators have measured fitness over the entire lifetime from seed to seed (e.g., Campbell et al. 2008), they have not generally tracked the fate of individual seeds in a way that allows for natural seed dispersion and spatially explicit interactions between seeds. 
In contrast, the gap between seeds and seedlings has been explored at the population level in studies of average fitness that do not assign offspring to particular parents (e.g., Ehrlén 2002; Bricker et al. 2010; Feldman and Morris 2011; Bricker and Maron 2012; Shefferson et al. 2014; see Clark et al. 2007 for review). In some cases, these studies have demonstrated density dependence in survival of seeds to seedlings, given natural seed dispersal. For example, in Ipomopsis aggregata, the species we address in this study, a higher production of seeds did not lead to an exactly equal increment in the numbers of seed offspring that reach reproductive maturity. We showed similar levels of population-wide density dependence both with an experiment in which we sowed seeds into plots and followed their fates en masse (Price et al. 2008) and in an observational demographic study in which we estimated seed rain in natural populations and related the numbers of plants that reach maturity to the density of this seed input (Waser et al. 2010).

Such studies at the level of entire populations cannot tell us, however, whether the relative fecundities of individuals suffice as a proxy for relative fitness at the individual level. The overall success of offspring might rise (or fall) across an entire population as a function of increasing average fecundity for the population in ways that do not exactly reflect effects of variation in individual parental fecundity. A variety of mechanisms could differentially influence individual fitness during the gap between generations (Metcalf and Pavard 2006). The novel mechanism we focus on is the spatial distribution of offspring and how it affects interactions between siblings. It is possible for the number of juveniles recruited to be independent of the number of seeds set in a population (extreme density dependence at the population level) and yet for an individual plant that sets twice as many seeds as its neighbor to contribute twice as many of those juveniles as its low-fecundity neighbor (absence of density dependence at the individual level). This situation could occur if the density of juveniles is constant over space but neighboring individuals overlap in dispersal of their seed offspring such that the high-fecundity parent has twice as many chances for a successful juvenile recruit. Conversely, an individual that sets twice as many seeds may not contribute twice as many offspring to the next generation if competition occurs primarily among siblings. The relative importance of sibling interactions and thus whether differences in seed production translate into proportional differences in overall fitness is likely to depend on how widely seed offspring are dispersed.

Here we address the question, Under what circumstances does making more seeds of similar size make an individual plant more fit in equal proportion? We address this question using a field experiment and a model of the spatial relationships of plants and their seed shadows. For the experiment, we sowed seeds of two known genotypes of $I$. ag- gregata into natural subalpine meadows in a fashion that mimicked overlapping seed shadows of pairs of neighboring plants, one with twice the fecundity of the other. We subsequently followed the success of seed offspring of each pair of parents over multiple years until the offspring had all died. The model in turn considers geometry of the seed shadows and assumes that offspring success is limited by a constant density of sites suitable for juvenile recruitment. The experiment revealed that recruitment of offspring from high- and low-fecundity parents occurred in an average ratio of $1.4: 1$ rather than $2: 1$ and that final total flower production in progenies of the two types of parents averaged $1.5: 1$, both of which agree well with the relative success of $1.42: 1$ predicted by the model. We then extend the model to consider spatial relationships of neighboring plants and their seed shadows other than those simulated by the experiment. We derive some general conclusions about how the spatial overlap of parental seed shadows affects the relationship between overall relative fitness and fecundity.

\section{Methods \\ Study System}

Ipomopsis aggregata Pursh (V. Grant) subsp. aggregata (Polemoniaceae) is a monocarpic perennial wildflower common in montane meadows of the western United States. It has been studied extensively near the Rocky Mountain Biological Laboratory (RMBL; 2,900-m elevation) in west central Colorado. In this area, the species generally flowers from late June through August, and seeds are shed from mid-August to mid-September. An individual plant can produce between 0 and 1,200 seeds (mean $=61$ seeds in Campbell 1989a). Seeds are gravity dispersed and fall close to the maternal parent. Most seedlings germinate after snowmelt in late May or early June of the year after they are shed. Plants grow for several years as vegetative rosettes and eventually flower once, set seed, and die; very few flower more than once, and those usually have been damaged as vegetative rosettes (Brody et al. 2007). Most surviving individuals flower in the third or fourth growing season after they germinate, but some take as long as a decade (Campbell 1997).

Previous demographic work with I. aggregata detected population-level density dependence at several life stages after seed germination. When seed input densities were varied experimentally over a 10-fold range from 33 to 333 seeds/ $\mathrm{m}^{2}$, seedling emergence increased linearly with seed input, but rosette size before flowering and flower production decreased with seed input density. As a result, the finite rate of population increase decreased nonlinearly with seed input density to a plateau at densities above about $125 \mathrm{seeds} / \mathrm{m}^{2}$ (Price et al. 2008). A study of natural variation in seed rain produced quantitatively similar nonlinear relationships due 
to density dependence after the germination stage in survival and flower production (Waser et al. 2010). At even higher seed input densities $\left(2,400\right.$ seeds $\left./ \mathrm{m}^{2}\right)$, density dependence was detected in survival to flowering for Ipomopsis aggregata ssp. candida by Juenger and Bergelson (2000).

\section{Experimental Approach}

Population-wide density dependence detected in these previous studies invites the question of whether the fitness of neighboring I. aggregata plants that produce different numbers of seeds is proportional to their fecundity. To explore this question, we sowed seeds of known genotypes into natural meadows to mimic partially overlapping seed shadows of pairs of maternal plants that differed twofold in fecundity. We then mapped emerging seedlings and, when those individuals (hereafter "recruits") were large enough, genotyped a sample of leaf material to assign parentage to the highfecundity mother, the low-fecundity mother, or to some other source. We censused the recruits annually to assess their survival and reproductive success.

Production of Known-Genotype Offspring. To produce offspring of known genotype, we collected flower bud tissue in 1999 from 52-61 bolting I. aggregata individuals growing within an area of 10-m radius in each of three meadows near the RMBL. We genotyped a total of 169 individuals based on alleles at the 6PGD-2 enzyme locus. Two alleles at this locus, designated "medium" and "slow," are present at high frequencies in populations of I. aggregata near the RMBL (Campbell 1991a) and are easily scored with starch-gel electrophoresis (Campbell 1991a; Campbell and Dooley 1992; Campbell et al. 2003). From the 169 genotyped individuals, we chose homozygous medium (MM) and homozygous slow (SS) individuals in each meadow that could be crossed to produce homozygous offspring. We potted these plants (23 MM and $17 \mathrm{SS}$ in total) and brought them into a screenhouse at the RMBL.

As new flowers opened on each plant and stigmas became receptive, we used a clean wooden toothpick to crosspollinate with pollen from two donors from the same meadow that had the same (homozygous) genotype, alternating which donor's pollen was applied first. We used multiple donors because Ipomopsis fruits are typically multiply sired (Campbell 1998). We did not emasculate recipient flowers, because $I$. aggregata is self-sterile (Waser and Price 1991). Plants were hand-pollinated and watered every other day and fertilized once per week using dilute (0.13\%) 20-20-20 NPK fertilizer. As fruits matured, seeds from each plant were collected and stored in coin envelopes.

Planting to Mimic High-and Low-Fecundity Parents. At the end of the 1999 flowering season, we selected from among the pool of sufficiently fecund parents two MM and two SS parents from each of two meadows and four of each genotype from the third meadow. We paired each MM with a sympatric SS parent to form eight MM-SS pairs. We originally used an additional meadow with two pairs of parents to generate a total sample of 10 pairs but later abandoned that meadow because seedling emergence was poor. Within each of the eight pairs, we assigned one plant to be high fecundity and the other to be low fecundity, and we counted out 300 and 150 seeds, respectively, from the envelopes for these two plants. The only exception was one pair in which the high-fecundity parent produced only 217 seeds; in that case, we used 109 seeds from the low-fecundity parent. MM and SS genotypes were equally represented in the highand low-fecundity treatments in each meadow. Because we assigned high- and low-fecundity treatments independently of the true fecundity of each parent, we avoided any trade-off between seed number and seed size. Seed mass was similar between the high- and low-fecundity treatments in any case (means across 10 pairs of parents $=1.13$ and $0.98 \mathrm{mg}$, respectively; randomized block ANOVA, $F_{1,9}=1.67, P=$ .23). We next established one or two pairs of plots within each meadow within $10 \mathrm{~m}$ of the cluster of source plants. Each $3.5 \times 3.0-\mathrm{m}$ plot was gridded with steel nails placed in the ground at $0.5-\mathrm{m}$ intervals. These reference nails allowed us to map and relocate I. aggregata plants within the plots. Existing natural vegetation within each plot was left intact except that after sowing seeds we removed flowering I. aggregata individuals from within each plot and $1.5 \mathrm{~m}$ beyond its perimeter in 1999 and 2000. Few seeds naturally fall beyond this distance, and dormancy of I. aggregata seeds is rare (1\%-4\%; Campbell 1997).

We sowed seeds from one pair of high- and low-fecundity parents into each plot, mimicking natural seed shadows from two plants that were $0.5 \mathrm{~m}$ apart in the center of the plot. This distance is a typical spacing for flowering individuals near the RMBL; nearest-neighbor distance averaged $0.37 \mathrm{~m}$ with a range of 0 (within measurement error) to $1.71 \mathrm{~m}$ in the central part of a population near one of our sites in 1987 (Campbell 1991a). We characterized natural seed shadows in two ways: by placing plastic sheets sprayed with sticky Tanglefoot (Tanglefoot, Grand Rapids, MI) around 12 flowering I. aggregata plants in 1995 and mapping the distribution of dispersed seeds, and by observing the spatial distribution of seedlings that emerged in 1997 around six isolated plants that had flowered in 1996. For plants on level ground, $<2 \%$ of seeds or seedlings were found $>1.5 \mathrm{~m}$ from the parent. Of the seeds or seedlings within $1.5 \mathrm{~m}$ of each parent, on average $53 \%$ were found within $0.5 \mathrm{~m}, 33 \%$ between 0.5 and $1.0 \mathrm{~m}$, and the remaining $14 \%$ between 1.0 and $1.5 \mathrm{~m}$. To mimic this pattern, for each parent in our experiment we scattered $53 \%$ of seeds evenly within a circular wire frame of radius $0.5 \mathrm{~m}$ centered 
on the imaginary location of that parent within a plot, $33 \%$ between frames of 0.5 - and 1.0 -m radius, and the remaining $14 \%$ between frames of $1.0-$ and $1.5-\mathrm{m}$ radius. Figure 1 shows the expected numbers of seeds from each parent that fell into various sectors of the overlapping seed shadows.

Offspring Censuses. Most I. aggregata seedlings that emerged in the seeded plots appeared in the spring of 2000, and a much smaller number appeared in 2001 . We returned every summer from 2000 to 2008 to census all individual recruits in the 2000 and 2001 seedling cohorts as well as preexisting individuals in the plots. To do this, we used $1-\mathrm{m}^{2}$ quadrats subdivided by fishing line into $10 \times 10-\mathrm{cm}$ squares and positioned them over the reference nails, allowing us to characterize the coordinates of each plant. In June of each year, when flowering individuals had begun to bolt, we recorded presence or absence of each mapped individual recruit, its stage (seedling, vegetative rosette, or flowering), and the size of each rosette (number of leaves and maximum leaf length).
At the end of each season in August, we recorded the cumulative number of flowers produced by those individuals that had flowered.

Seedlings could not be genotyped immediately on emergence, since sampling tissue required removing one or two leaves. Instead, we waited up to 2 years until plants from the 2000 and 2001 seedling cohorts had successfully established and had formed sizable rosettes that could withstand removal of leaf tissue. We genotyped each recruit at least twice. A plant was assigned to the high- or low-fecundity treatment only if there was agreement in gel scores and if the genotype was MM or SS.

In all, we successfully genotyped 245 recruits. Of these, 185 were either MM or SS genotypes and 60 were other genotypes that could not be assigned to one of the two parents whose seeds we had sown. This large number of "impossible" genotypes was unexpected for two reasons. First, we had removed all flowering stalks within each plot and for $1.5 \mathrm{~m}$ around it in 1999 and 2000, under the assumption

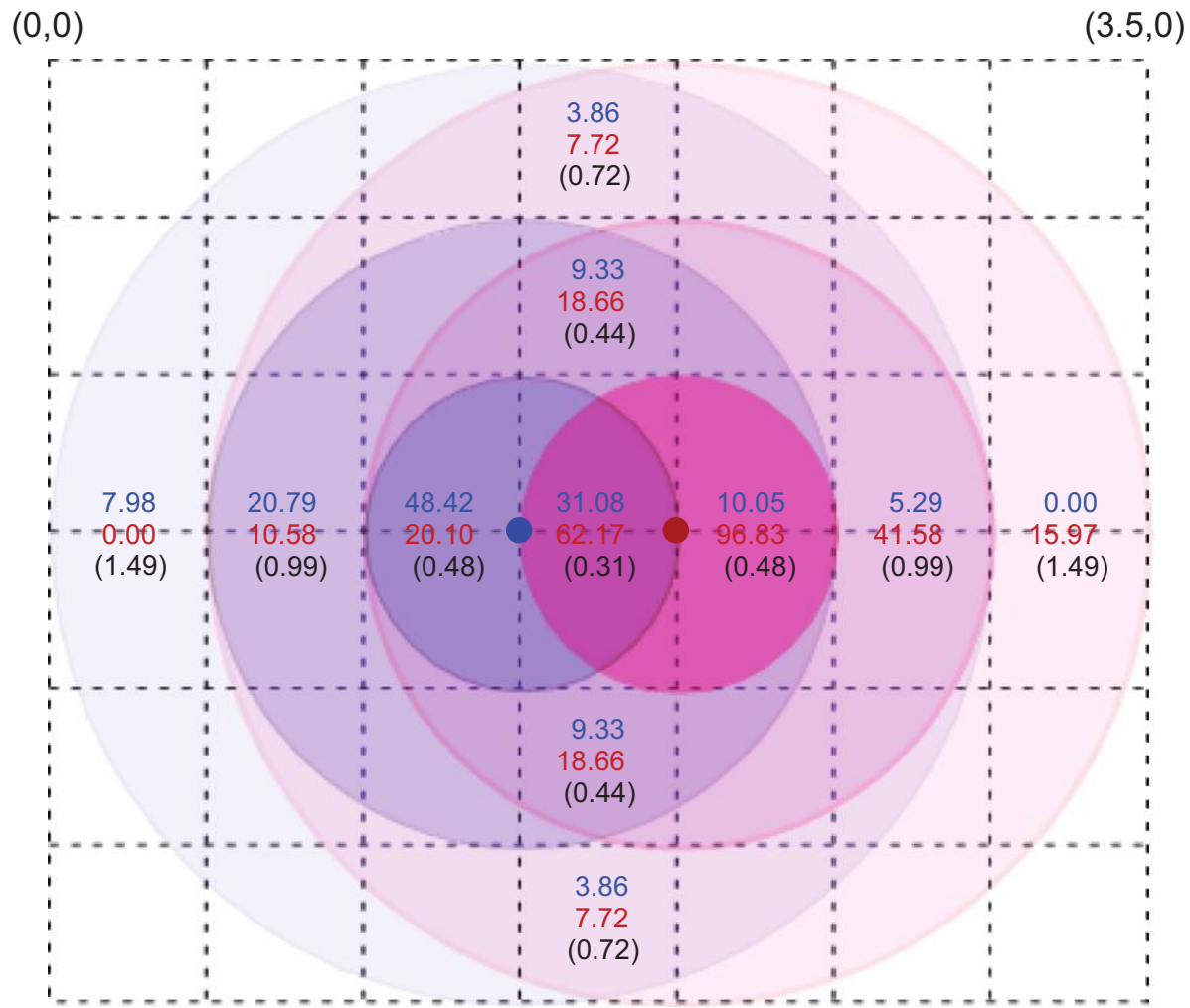

$(0,3.0)$

Figure 1: Diagram of the experimental seed-sowing experiment, showing the expected numbers of seeds from high-fecundity (red) and lowfecundity (blue) parents that would land in each of 11 sectors within overlapping seed shadows of the parents, assuming uniform distribution of seeds within each annulus of a given seed shadow. The sown plots are $3.5 \mathrm{~m}$ wide and $3.0 \mathrm{~m}$ tall; the origin $(0,0)$ is in the upper left corner of each plot, and the positions of hypothetical high-fecundity (red dot) and low-fecundity (blue dot) seed parents are $(x=2.0, y=1.5)$ and $(x=1.5, y=1.5)$, respectively. The total area of each parent's seed shadow is $7.0686 \mathrm{~m}^{2}$, and the areas (in square meters) of each overlapping and nonoverlapping sector of the two seed shadows are given in black in italics within parentheses. 
that by doing so we were eliminating virtually all seed dispersal into the plots. Second, previous studies indicated that very few seeds of I. aggregata remain dormant in the soil beyond the first summer after they are shed. Regardless of the cause, the existence of these genotypes indicates some seed flow into the plots or some unusual dormancy. These volunteer seeds in turn imply that we could have wrongly assigned some MM or SS individuals as offspring of our experimental parents when in fact they came from other sources, representing what we term "cryptic seed flow." We therefore corrected the numbers of individuals assigned to experimental parents to account for cryptic seed flow (calculations in app. A; apps. A-C are available online).

From data on the life histories of offspring assigned to each parent, we then determined the number of seed offspring that recruited (germinated and survived to be genotyped), the number that survived to flower, and their fecundity (estimated as the number of flowers they ultimately produced). These numbers were calculated with and without the correction for cryptic seed flow. We then compared each of these measures of offspring success against expectations from two models for the relative success of highand low-fecundity neighbors - one model that assumes no density dependence and an alternative model that assumes a simple, "safe site" form of density dependence as described in the next section.

\section{Models}

Expected Relative Offspring Success under Two Alternative Models. If there were no density dependence, we would expect each seed to have equal success in establishment, survival, and flowering, in which case the high-fecundity parents in our experiment would produce on average twice the number of successful offspring as the low-fecundity parents. Alternatively, suppose that there is a uniform density of safe sites suitable for seed germination and recruitment of juvenile plants throughout the area in which seeds fall and that only one juvenile can occupy each safe site. Under this simple density-dependent model, the numbers of successful offspring in various sectors of the seed shadows of two parents (fig. 1) would be proportional to the areas of those sectors. The expected ratio of successful offspring from the highversus low-fecundity parent would be less than $2: 1$ because offspring of the high-fecundity parent experience greater competition with siblings than do offspring of the lowfecundity parent. At one extreme where seed shadows do not overlap at all, both parents would have the same number of successful progeny. Given the actual geometry of seed shadows used in the experiment, this model predicts a $1.42: 1$ overall ratio of successful offspring from high- versus lowfecundity parents (derivation in app. B, sec. 1).
Extension of the Spatial Safe Site Model. We extended the spatial model in three ways. First, we explored the effect of seed dispersal, by comparing results under the pattern of seed dispersal actually observed for I. aggregata to results with highly localized seed dispersal and with even dispersion over the full seed shadow with a radius of $1.5 \mathrm{~m}$ (app. B, sec. 2). Second, we considered effects of the distance between adult plants relative to the size of their combined seed shadows (app. B, sec. 3). Third, we explored the effect of including more than two parents, by allowing for more than two overlapping seed shadows (app. B, sec. 4; figs. B1, B2; figs. B1, B2, C1 are available online).

\section{Statistical Comparison of Experimental Results with Model Predictions}

We tested for density dependence in offspring success by comparing recruits per seed input and number of flowers produced per recruit between the high- and low-fecundity treatments. Since each of the eight plots was sown with seeds from one high- and one low-fecundity parent, we used a randomized block analysis of variance with parent fecundity as a fixed effect and plot as a random effect (PROC MIXED in SAS ver. 9.3). Residuals for both dependent variables were normally distributed based on Shapiro-Wilks tests. Density dependence of survival from recruit to flowering was also tested by using the $\mathrm{R}$ (ver. 3.3.1) function nnd to determine the distance to the nearest neighbor and then using a logistic regression with a binomial distribution to test for the effect of nnd on survival (PROC GENMOD in SAS ver. 9.3).

To compare experimental results with model predictions, we calculated the ratio of recruits produced by the high- and low-fecundity parents for each plot and used the eight values to determine the standard error around the mean relative fitness for comparison with the expected value of 1.42 from the model described in appendix B, section 1 . The ratios were calculated in two ways: (1) assuming that all of the MM and SS recruits were indeed offspring of the experimental parents and (2) incorporating cryptic seed flow. For the latter, we calculated cryptic seed flow rates separately by meadow and subtracted the appropriate rate from the observed offspring success in each plot. We also regressed the number of recruits from the high-fecundity parent on the number of recruits from the low-fecundity parent in the same plot and tested the linear hypothesis that the slope is equal to 2 and the linear hypothesis that the slope is equal to 1.42. Ratios of successful recruits were also calculated for total survivors to flowering and for total flowers produced by offspring of the two types of parents.

\section{Results}

From the 3,476 seeds sown into the eight plots, a total of 185 seedlings emerged in 2000 or 2001 and survived to be 
genotyped as MM or SS 1-2 years later. Data are available in the Dryad Digital Repository: http://dx.doi:10.5061/dryad .6q023 (Campbell et al. 2017). Spatial maps of these recruits are shown in figure $\mathrm{C} 1$. This number corresponds to an overall recruitment of 5.3\%. For genotyped offspring, percent recruitment was higher on average for seeds from low- than from high-fecundity parents (mean $=6.9 \%$ vs. $4.4 \%$; randomized block ANOVA on proportion, $F_{1,7}=8.83, P=$ $.0208)$. Out of the 185 recruits, 47 plants survived to flower. Age at flowering ranged from 2 to 7 years with a mean of 3.7 years. The probability of surviving to flower did not depend significantly on distance to the nearest neighbor plant (logistic regression slope of logit on distance $=0.0215$; Wald $\left.\chi^{2}=2.68, P=.1017\right)$. Furthermore, the number of flowers eventually produced per recruit, a statistic that combines survival to flowering and number of flowers per survivor, did not differ detectably between low- and high-fecundity parents (means $=17.4$ vs. $10.8 ; F_{1,7}=2.17, P=.1839$ ). Thus, there was no strong evidence for additional density-dependent effects among siblings beyond the stage of seedling establishment.

Accordingly, the high-fecundity parents did not produce twice as many successful offspring as the low-fecundity parents. The actual ratio for recruits with SS or MM genotype averaged 1.40:1 across the eight plots (fig. 2). This ratio was 1.31:1 (80 low- and 105 high-fecundity recruits) when we

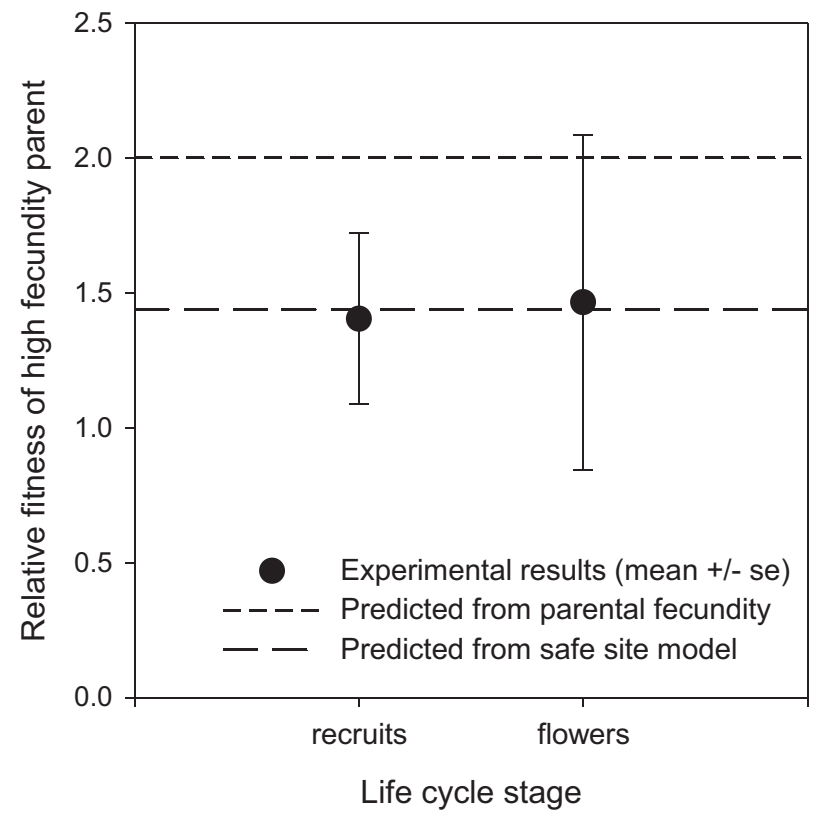

Figure 2: Actual relative fitness of the high-fecundity parent shown as mean and standard error across eight sites and compared with predicted values based only on parental fecundity or the safe site model. Results are based on number of recruits that survived to a size at which we could genotype them (ignoring cryptic seed flow) and total flowers produced by these offspring. summed the total numbers of recruits across all plots rather than averaging ratios across plots.

Cryptic seed flow ranged from $7.0 \%$ to $19.7 \%$ across the three meadows (app. A). Subtracting recruits at those rates from the observed numbers of MM and SS recruits in each plot caused us to attribute 26 recruits (13 of each treatment) to cryptic seed flow. Taking cryptic seed flow into account changes the overall fitness ratio only modestly, from $1.31: 1$ to a new value of $1.37: 1$.

These observed measures for components of relative fitness are all very close to the predicted value of $1.42: 1$ under the model that assumes a fixed density of safe sites and that duplicates the size of seed shadows used in the experiment (fig. 1; app. B, sec. 1). Indeed, a regression of recruits from the high-fecundity parent on recruits from the low-fecundity parent produces a slope indistinguishable from $1.42(P=$ $.616)$ and different from $2.0(P=.0002)$. As the model illustrates, it is only in the central portions of the overlapping seed shadows (those sectors that symmetrically overlap the midline between the positions of the parents) that the expected ratio of seeds from the high- and low-fecundity parents is equal to $2: 1$. In outer sectors of the seed shadows, the expected ratio deviates from $2: 1$. Thus, the relative strength of sibling competition increases for each parent in sectors skewed toward its side of the midline but is higher overall for the high-fecundity parent. Sibling competition for limited safe sites ameliorates parental fecundity advantage, reducing it from $2: 1$ to $1.42: 1$. The relatively small numbers of recruits prevented us from doing a finer-scale comparison with model predictions on a sector by sector basis.

The ratio of survivors to flowering from high- versus lowfecundity parents averaged $1.1: 1$, and the final ratio of total flowers from high- versus low-fecundity parents, a more complete assessment of overall success that includes both parental fecundity and offspring success, averaged 1.5:1 (fig. 2). Both of these latter ratios showed high variation due to the small sample size of offspring that survived to flower. The ratio of total flowers produced was indistinguishable from either $1.42: 1$ or $2.0: 1(P>.05)$, the contrasting expectations under the model that assumes a fixed density of safe sites and hence density dependence versus the model that assumes no density dependence.

Extending the density-dependent spatial model showed that sibling competition for safe sites is more severe as seed dispersal becomes more localized and as the relative distance between the parents increases. Those conditions lead to lower ratios of offspring success from a high- than a lowfecundity parent (fig. 3). At the extreme, when seed shadows do not overlap at all, as long as the number of recruits remains proportional to the area, a high-fecundity parent has the same fitness as a low-fecundity parent, despite producing more seeds (fig. 3). At the other extreme, when seed shadows are completely overlapping, fitness is expected to 


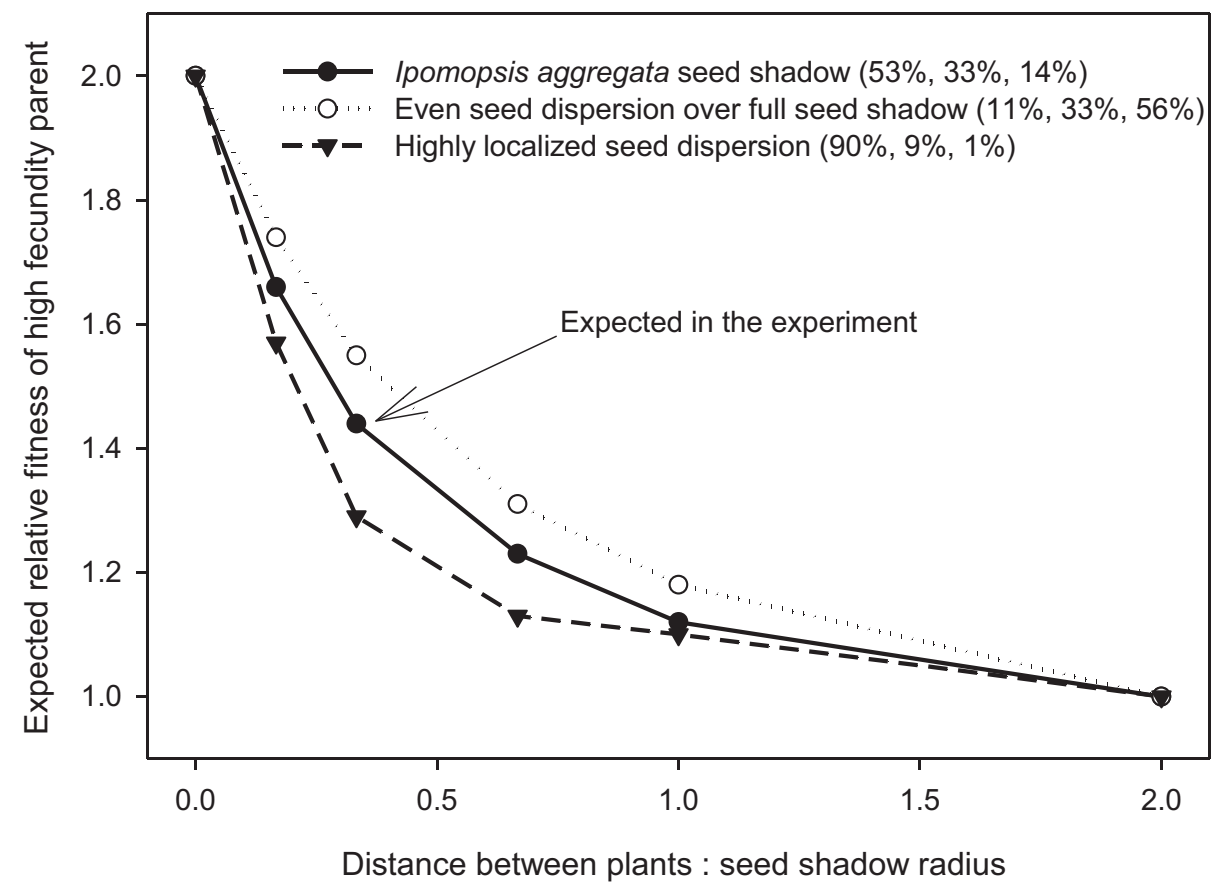

Figure 3: Results of the generalized spatial model of competition for safe sites. Relative fitness of the high-fecundity parent is plotted against the distance between adult plants compared to the radius of the entire seed shadow. Three types of seed shadows are shown: (i) as estimated for Ipomopsis aggregata for the three distance classes, (ii) seeds dispersed evenly over the entire seed shadow regardless of distance class, and (iii) highly localized seed dispersal.

be proportional to fecundity. As seed shadows move from nonoverlapping to completely overlapping, a parent with twice the fecundity of another experiences an accelerating increase in relative fitness from $1: 1$ to $2: 1$ compared to the low-fecundity parent, even though its absolute fitness declines by one-third due to sharing a fixed number of safe sites for recruitment. If a parent has a seed shadow overlapping partially with multiple seed shadows instead of only one other seed shadow, relative fitness is expected to more closely follow relative fecundity as the offspring experience less sibling competition (fig. B2).

\section{Discussion}

Many studies of selection on reproductive traits use fecundity as an estimate of fitness, but the extent to which an individual fitness component such as fecundity correlates with overall fitness is rarely investigated (Kingsolver et al. 2012). That the assumption of direct proportionality between fecundity and longer-term fitness can fail was pointed out for plant-herbivore interactions by Harper (1977), who stressed that density-dependent processes, such as safe site limitation, could modify the differential success of individuals measured at the time of reproduction. Here we showed that individual differences in plant fecundity, such as those that depend on a floral trait, can be partially offset by postdispersal density de- pendence, so that overall fitness need not be exactly proportional to seed set. In our experiment, successful per-capita offspring recruitment was higher on average for seeds from low-fecundity Ipomopsis aggregata parents, and a twofold difference in seed set translated into a smaller difference (1.4to 1.5 -fold) in established vegetative rosettes and offspring flower production.

Previous studies showed that population growth rate is linked to seed production in multiple species, including I. aggregata (Price et al. 2008). To our knowledge, however, the results presented here are the first study of links between seed production, offspring survival, and reproduction at the level of individuals within populations of plants with overlapping seed shadows under natural conditions. A populationlevel approach can tell us how sensitive population growth rate is to fecundity versus survival and how a decline in pollination or an increase in seed predation may affect the persistence of a particular plant species (Weber and Kolb 2011; Castro et al. 2015). But a population-level approach cannot tell us whether individual fecundity is necessarily an accurate measure of individual fitness, which is critical to an unbiased estimate of selection.

Our spatial model predicted that individual differences in fecundity can be partially offset by sibling competition, and our field experiment showed good agreement with the quantitative prediction from a spatial model that included 
the particulars of spacing between parents and extent of seed dispersal. The experiment showed that such modifications of fitness can happen, but two caveats are in order. The first is that a limited and uniform distribution of safe sites for offspring recruitment is not the only mechanism of density dependence that could operate in the I. aggregata system. The density dependence could be driven by competition for renewable resources, in which case its magnitude could change as resources become sufficiently abundant to allow all seeds to survive, rather than by physical characteristics of specific microsites for seedlings. In principle, density-dependent attack by shared enemies such as herbivores or pathogens could also yield a similar outcome. Predispersal density dependence in the form of a trade-off between seed number and seed size is theoretically possible but was not detected at the maternal plant level in a previous study (Waser et al. 1995). The second caveat is that the exact quantitative result we obtained may or may not be a good representation for natural populations of the species. We attempted to choose a realistic spacing of $0.5 \mathrm{~m}$ between parents, but actual spacing can vary from 0 to $1.7 \mathrm{~m}$ (Campbell 1991a), and the quantitative effect of sibling competition is sensitive to spacing (fig. 3), other aspects of the spatial geometry of the populations, and genetic diversity of seeds, as argued below. Furthermore, density-dependent effects on offspring success could vary with temporal variation in environmental conditions. Here we saw strong effects on survival of recruits to establishment for seeds that germinated in 2000 or 2001, whereas in an earlier study of seeds that germinated in 1996 we saw a density-dependent effect on the number of flowers produced when offspring of these seeds matured but not on survival to flowering (Waser et al. 2010). As our populationlevel studies have never detected density dependence at the seedling germination stage (Waser et al. 2010), it is likely that the density dependence we witnessed here occurred between germination and establishment of the recruits.

Changing a $2: 1$ ratio in fitness to a $1.5: 1$ ratio could have a large impact on the rate of evolution because it reduces the strength of natural selection by one-third. For a scenario of a single gene trait and complete dominance with two phenotypes corresponding to high- and low-fecundity parents, the selection coefficient would change from 0.5 to 0.33 . At a point where the allele frequencies are equal at 0.5 , including the effects of postdispersal density dependence would cause the deleterious allele frequency to be 0.045 less rather than 0.071 less in the next generation (following eq. [3.6b] in Hedrick 2000). A similar reduction by one-third holds for a standardized linear selection differential on a quantitative trait, assuming that the density dependence is linear. As one example from I. aggregata, plants that bloomed earlier in 1986 produced more seeds, and incorporating the density dependence found here would reduce the standardized selection differential through female function from -0.44
(Campbell 1991b) to -0.29 if we ignore the caveats in the preceding paragraph. Selection through male function is likely to be less affected by postdispersal density dependence, as pollen from a given individual is typically spread among many seed-producing mates (Campbell 1989b, 1998). Thus, the effects of sibling interactions on selection should be greatest for traits that are under selection through female function only, such as the proportion of time that I. aggregata flowers spend in the female phase (Campbell 1989b).

Our generalized model illustrates conditions under which fitness estimates based only on fecundity are likely to be inaccurate because of postdispersal processes. First, offspring success must be density dependent. Second, seed dispersal must be limited so that siblings interact with each other. Furthermore, for interactions to be stronger within rather than among sibships, neighboring parents need to be far enough apart that their seed shadows do not completely overlap. The degree to which fecundity differences determine relative fitness of parents in our model erodes rapidly and nonlinearly as the overlap between parental seed shadows decreases, eventually disappearing completely when seed shadows do not overlap at all (fig. 3). The model also shows that the fecundity advantage erodes more quickly as seed dispersal becomes more localized. These effects are lessened, however, when parental seed shadows overlap with those of more neighboring plants.

In addition to the geometry of seed shadows, we could expect the relative strength of sibling interactions to depend on the level of genetic diversity of seeds produced by a single parent. Genetic diversity can decrease sibling interactions through a sampling effect, niche partitioning, or decreased enemy sharing (Price and Waser 1982; Hughes et al. 2008). For example, in Eucalyptus, offspring fitness increased with the incidence of multiple paternity (Breed et al. 2014). Ipomopsis aggregata exhibits high multiple paternity, with a single fruit averaging four pollen donors (Campbell 1998), more than the two pollen donors we used for each experimental plant in this study. The number of donors at the plant level is likely to be even higher. Thus, sibling interactions may be weaker than those we infer to have occurred in our experiment, which would tend to ameliorate the relative strength of sibling competition compared to nonsibling competition and make relative fitness resemble fecundity differences more closely. Multiple paternity is quite high in Ipomopsis compared to other species that have been studied (reviewed in Mitchell et al. 2013), suggesting that effects of sibling interaction may be more important for other plant species with lower pollen carryover (and hence lower multiple paternity), at least those that have no specialized means of longer-distance seed dispersal.

Overall, female fitness differences based on fecundity would be most likely reduced by sibling interactions in plant populations with (1) low adult density, (2) low seed dispersal, 
and (3) little multiple paternity. If sibling interactions are facilitative rather than competitive, fitness differences could be magnified rather than reduced by seed shadow overlap. Such positive interactions have been reported in situations where neighboring plants ameliorate a stressful environment (Butterfield and Callaway 2013)_for example, by acting as nurse plants that provide shade or by facilitating attractiveness to pollinators. Consider the frequently correlated traits of plant height and flower number. Higher flower number of a parent often results in more seeds (Harder and Johnson 2009), and the resulting seedlings, if they inherit taller stature, are likely to better shade one another, reducing water loss and perhaps increasing survival. In such a scenario, patterns in offspring survival could enhance fitness differences seen in seed set. Taller plants that produce more flowers might also disperse seeds farther, as is seen across species for wind-dispersed seeds (Bullock et al. 2017). If so, we would expect a negative correlation between fecundity and the relative strength of sibling interactions, which could impose selection favoring traits that increase dispersal if sibling interactions are primarily competitive or traits that decrease dispersal if sibling interactions are facilitative.

In conclusion, our model and field experiment show that the fitness benefit of high fecundity may be altered by densitydependent offspring success, such that relative fecundity may not be a completely accurate measure of overall fitness. We recognize that the use of genetic markers to measure offspring success and overall plant fitness directly is not widely practical. Still, future investigators of selection on plant reproductive traits may wish to consider the likelihood that interactions among siblings could alter fitness based solely on fecundity, especially if they are studying a system that combines low adult density, restricted seed dispersal, and low genetic diversity of seeds. Extending theoretical models to consider an even wider range of conditions for fitness modification due to spatial distribution of parents and offspring dispersal would also be well worthwhile.

\section{Acknowledgments}

For assistance in field and laboratory we thank Ruben Alarcón, Heidi Albright, Regula Bollier, Xavier Colleau, Andreas Erhardt, Dawn Graydon, Ben Koch, Ursula Lang, Denise Massart, Margie Mayfield, Twila Paterson, Gregory Pederson, Alta Price, Brook Peterson, Jessica Ruvinsky, Anne Shrag, Anne Valdenaire, and Carrie Wu. We thank Christina Caruso, John Powers, Wilnelia Recart, Stephen Weller, Alice Winn, and two anonymous reviewers for comments on the manuscript. We are grateful to the Rocky Mountain Biological Laboratory for logistical support and the National Science Foundation (grant DEB-9805034) for financial support.

\section{Literature Cited}

Barrett, S. C. H., and L. D. Harder. 1996. Ecology and evolution of plant mating. Trends in Ecology and Evolution 11:73-79.

Breed, M. F., M. J. Christmas, and A. J. Lowe. 2014. Higher levels of multiple paternities increase seedling survival in the long-lived tree Eucalyptus gracilis. PLoS ONE 9:e90478.

Bricker, M., and J. Maron. 2012. Postdispersal seed predation limits the abundance of a long-lived perennial forb (Lithospermum ruderale). Ecology 93:532-543.

Bricker, M., D. Pearson, and J. Maron. 2010. Small-mammal seed predation limits the recruitment and abundance of two perennial grassland forbs. Ecology 91:85-92.

Brody, A. K., M. V. Price, and N. M. Waser. 2007. Life-history consequences of vegetative damage in scarlet gilia, a monocarpic plant. Oikos 116:975-985.

Bullock, J. M., L. M. González, T. Tamme, L. Götzenberger, S. M. White, M. Pärtel, and D. A. P. Hooftman. 2017. A synthesis of empirical plant dispersal kernels. Journal of Ecology 105:6-19.

Butterfield, B. J., and R. M. Callaway. 2013. A functional comparative approach to facilitation and its context dependence. Functional Ecology 27:907-917.

Campbell, D. R. 1989a. Inflorescence size: test of the male function hypothesis. American Journal of Botany 76:730-738.

. 1989b. Measurements of selection in a hermaphroditic plant: variation in male and female pollination success. Evolution 43:318334.

. 1991a. Comparing pollen dispersal and gene flow in a natural population. Evolution 45:1965-1968.

- 1991b. Effects of floral traits on sequential components of fitness in Ipomopsis aggregata. American Naturalist 137:713-737.

. 1997. Genetic and environmental variation in life-history traits of a monocarpic perennial: a decade-long field experiment. Evolution 51:373-382.

. 1998. Multiple paternity in fruits of Ipomopsis aggregata (Polemoniaceae). American Journal of Botany 85:1022-1027.

Campbell, D. R., R. Alarcón, and C. A. Wu. 2003. Reproductive isolation and hybrid pollen disadvantage in Ipomopsis. Iournal of Evolutionary Biology 16:536-540.

Campbell, D. R., A. K. Brody, M. V. Price, and G. Aldridge. 2017. Data from: Is plant fitness proportional to seed set? an experiment and a spatial model. American Naturalist, Dryad Digital Repository, http://dx.doi.org/10.5061/dryad.6q023.

Campbell, D. R., and J. L. Dooley. 1992. The spatial scale of genetic differentiation in a hummingbird-pollinated plant: comparison with models of isolation by distance. American Naturalist 139:735-748.

Campbell, D. R., N. M. Waser, G. Aldridge, and C. A. Wu. 2008. Lifetime fitness in two generations of Ipomopsis hybrids. Evolution 62: 2616-2627.

Castro, S., T. Dostalek, S. van der Meer, G. Oostermeijer, and Z. Munzbergova. 2015. Does pollen limitation affect population growth of the endangered Dracocephalum austriacum L.? Population Ecology 57:105-116.

Clark, C. J., J. R. Poulsen, D. J. Levey, and C. W. Osenberg. 2007. Are plant populations seed limited? a critique and meta-analysis of seed addition experiments. American Naturalist 170:128-142.

Conner, J. K., S. Rush, S. Kercher, and P. Jennetten. 1996. Measurements of natural selection on floral traits in wild radish (Raphanus raphanistrum). II. Selection through lifetime male and total fitness. Evolution 50:1137-1146. 
Ehrlén, J. 2002. Assessing the lifetime consequences of plant-animal interactions for the perennial herb Lathyrus vernus (Fabaceae). Perspectives in Plant Ecology, Evolution, and Systematics 5:145-163.

Feldman, T. S., and W. F. Morris. 2011. Higher survival at low density counteracts lower fecundity to obviate Allee effects in a perennial plant. Journal of Ecology 99:1162-1170.

Harder, L. D., and S. D. Johnson 2009. Darwin's beautiful contrivances: evolutionary and functional evidence for floral adaptation. New Phytologist 183:530-545.

Harper, J. L. 1977. The population biology of plants. Academic Press, London.

Hedrick, P. W. 2000. Genetics of populations. 2nd ed. Jones \& Bartlett, Sudbury, MA.

Hughes, A. R., B. D. Inouye, M. T. J. Johnson, N. Underwood, and M. Vellend. 2008. Ecological consequences of genetic diversity. Ecology Letters 11:609-623.

Juenger, T., and J. Bergelson. 2000. Factors limiting rosette recruitment in scarlet gilia, Ipomopsis aggregata: seed and disturbance limitation. Oecologia 123:358-363.

Kessler, D., D. Diezl, D. G. Clark, T. A. Colquhoun, and I. T. Baldwin. 2013. Petunia flowers solve the defence/apparency dilemma of pollinator attraction by deploying complex floral blends. Ecology Letters 16:299-306.

Kingsolver, J. G., S. E. Diamond, A. M. Siepielski, and S. M. Carlson. 2012. Synthetic analyses of phenotypic selection in natural populations: lessons, limitations and future directions. Evolutionary Ecology 26:1101-1118.

McGraw, J. B., and H. Caswell. 1996. Estimation of individual fitness from life-history data. American Naturalist 147:46-64.

Meléndez-Ackerman, E. J., and D. R. Campbell. 1998. Adaptive significance of flower color and inter-trait correlations in an Ipomopsis hybrid zone. Evolution 52:1293-1303.

Metcalf, C. J. E., and S. Pavard. 2006. Why evolutionary biologists should be demographers. Trends in Ecology and Evolution 22:205-212.

Mitchell, R. J., W. G. Wilson, K. G. Holmquist, and J. D. Karron. 2013. Influence of pollen transport dynamics on sire profiles and multiple paternity in flowering plants. PLoS ONE 8:e76312.

Mojica, J. P., and J. K. Kelly. 2010. Viability selection prior to trait expression is an essential component of natural selection. Proceedings of the Roval Society B 277:2945-2950.

Moorad, J. A. 2014. Individual fitness and phenotypic selection in age-structured populations with constant growth rate. Ecology 95:1087-1095.
Price, M. V., D. R. Campbell, N. M. Waser, and A. K. Brody. 2008 Bridging the generation gap in plants: pollination, parental fecundity, and offspring demography. Ecology 89:1596-1604.

Price, M. V., and N. M. Waser. 1982. Population structure, frequencydependent selection, and the maintenance of sexual reproduction. Evolution 36:35-43.

Shaw, R. G., C. J. Geyer, S. Wagenius, H. H. Hangelbroek, and J. R. Etterson. 2008. Unifying life-history analyses for inference of fitness and population growth. American Naturalist 172:E35-E47.

Shefferson, R. P., R. J. Warren II, and H. R. Pulliam. 2014. Life-history costs make perfect sprouting maladaptive in two herbaceous perennials. Journal of Ecology 102:1318-1328.

Stanton, M. L., A. A. Snow, and S. N. Handel. 1986. Floral evolution-attractiveness to pollinators increases male fitness. Science 232:1625-1627.

Strauss, S. Y., and J. B. Whittall. 2006. Non-pollinator agents of selection on floral traits. Pages 120-138 in L. Harder and S. Barrett, eds. Ecology and evolution of flowers. Oxford University Press, Oxford.

Waser, N. M., D. R. Campbell, M. V. Price, and A. K. Brody. 2010. Density-dependent demographic responses of a semelparous plant to natural variation in seed rain. Oikos 119:1929-1935.

Waser, N. M., and M. Price. 1991. Reproductive costs of self-pollination in Ipomopsis aggregata (Polemoniaceae): are ovules usurped? American Journal of Botany 78:1036-1043.

Waser, N. M., R. G. Shaw, and M. V. Price. 1995. Seed set and seed mass in Ipomopsis aggregata: variance partitioning and inferences about postpollination selection. Evolution 49:80-88.

Weber, A., and A. Kolb. 2011. Evolutionary consequences of habitat fragmentation: population size and density affect selection on inflorescence size in a perennial herb. Evolutionarv Ecology 25:417428.

\section{References Cited Only in the Online Appendixes}

Devlin, B., and N. C. Ellstrand. 1990. The development and application of a refined method for estimating gene flow from angiosperm paternity analysis. Evolution 44:248-259.

Associate Editor: Christina M. Caruso Editor: Alice A. Winn 